\title{
Neuroprotective mechanisms of $S$-allyl-L-cysteine in neurological disease (Review)
}

\author{
YASUHIRO KOSUGE
}

\begin{abstract}
Laboratory of Pharmacology, School of Pharmacy, Nihon University, Funabashi-shi, Chiba 274-8555, Japan
\end{abstract}
Received July 23, 2019; Accepted August 26, 2019

DOI: $10.3892 / \mathrm{etm} .2019 .8391$

\begin{abstract}
S$-allyl-L-cysteine (SAC) is a sulfur-containing amino acid present in garlic and exhibits a wide range of biological activities such as antioxidant, anti-inflammatory, and anticancer agent. An earlier study demonstrated that SAC ameliorates oxidative damage in a model of experimental stroke. However, the antioxidant property of SAC does not suffice to explain its beneficial effects in terms of the underlying mechanisms. Endoplasmic reticulum (ER) stress and ER stress-induced cell death have been shown to be involved in various neurological diseases such as brain ischemia, Alzheimer's disease, Parkinson's disease, amyotrophic lateral sclerosis and Huntington's disease. We have previously demonstrated that SAC exerts significant protective effects against ER stress-induced neurotoxicity in cultured rat hippocampal neurons and organotypic hippocampal slice cultures.
\end{abstract}

Correspondence to: Dr Yasuhiro Kosuge, Laboratory of Pharmacology, School of Pharmacy, Nihon University, 7-7-1 Narashinodai, Funabashi-shi, Chiba 274-8555, Japan

E-mail: kosuge.yasuhiro@nihon-u.ac.jp

Abbreviations: AGE, aged garlic extract; ASK-1, apoptosis signal-regulating kinase $1 ; \mathrm{A} \beta$, amyloid $\beta$-peptide; boc, tert-butyl carbamate group; $\mathrm{CHOP}, \mathrm{C} / \mathrm{EBP}$ homologous protein; ent, enantiomeric; ent-SAC, S-allyl-D-cysteine; ent-SBC, $S$-benzyl-D-cysteine; ER, endoplasmic reticulum; GADD153, growth arrest and DNA damage-inducible protein 153; GSAC, $\gamma$-glutamyl-S-allyl-cysteine; IRE1, inositol-requiring enzyme 1; MCAO, middle cerebral artery occlusion; MOANBS, methyl $O$-allyl- $N$-boc-L-serinate; MOAS, methyl $O$-allyl-L-serinate; MPTP, 1-methyl-4-phenyl-1,2,3,6-tetrahydropyridine; MSAC, methyl $S$-allyl-L-cysteinate; NASAC, $N$-acetyl- $S$-allyl-L-cysteine; NASACA, $N$-acetyl- $S$-allyl-L-cysteine amide; NGF, nerve growth factor; OAS, $O$-allyl-L-serine; OASA, $O$-allyl-L-serine amide; rac, racemic; rac-SAHC, $S$-allyl-DL-homocysteine; SAC, $S$-allyl-L-cysteine; SACA, $S$-allyl-L-cysteine amide; SBC, $S$-benzyl-L-cysteine; SCMC, S-carboxymethyl-L-cysteine; SEC, S-ethyl-L-cysteine; SMC, $S$-methyl-L-cysteine; SPC, $S$-propyl-L-cysteine; SPNC, $S$-propynyl-L-cysteine; STC, $S$-trityl-L-cysteine; TRAF2, tumor necrosis factor receptor-associated factor 2; UPR, unfolded protein response; $\gamma \mathrm{GTP}, \gamma$-glutamyl transpeptidase

Key words: S-allyl-L-cysteine, calpain, endoplasmic reticulum stress, neuroprotection, $\mathrm{Ca}^{2+}$, cysteine-containing compounds
Recently, we demonstrated that these results are due to the direct suppression of calpain activity via the binding of SAC to this enzyme's $\mathrm{Ca}^{2+}$-binding domain. We also found that the protective effects of the side-chain-modified SAC derivatives, $S$-ethyl-L-cysteine (SEC) and $S$-propyl-L-cysteine (SPC), against ER stress-induced neurotoxicity were more potent than those of SAC in cultured rat hippocampal neurons. In addition, SAC, SEC and SPC have been shown to decrease the production of amyloid- $\beta$ peptide in the brains of mice with D-galactose-induced aging. These three hydrophilic cysteine-containing compounds have also been shown to exert neuroprotective effects against dopaminergic neuron injury in a murine model of Parkinson's disease induced by 1-methyl-4-phenyl-1,2,3,6-tetrahydropyridine (MPTP). In this review, we aim to provide a current overview of the protective actions of SAC and the SAC-related compounds, SEC and SPC, in neurodegenerative disease and discuss the promise of $\mathrm{SAC}$ as a prototype for developing novel therapeutic drugs for neurological diseases.

\section{Contents}

1. Introduction

2. Protective effects of SAC against neuronal death due to ER stress

3. Calpain is a probable target molecule for SAC

4. Analogs of SAC

5. Conclusions and future perspectives

\section{Introduction}

$S$-allyl-L-cysteine (SAC) is the most abundant organosulfur compound derived from garlic (Allium sativum) and is biosynthesized by hydrolysis of $\gamma$-glutamyl-S-allyl-cysteine (G SAC) by the enzyme $\gamma$-glutamyl transpeptidase ( $\gamma$ GTP). SAC is known as a water soluble bioactive compound of extremely high antioxidant capacity, and has long been used as a common dietary supplement and in traditional medicine (1). Moreover, SAC has been reported to exert multiple biological effects, such as anti-diabetic (2), cholesterol-lowering (3), anticancer (4) and anti-hepatotoxic (5) effects. SAC and other organosulfur compounds are found in aged garlic extract (AGE), which is produced by natural extraction from fresh garlic followed by 
incubation for $>10$ months in aqueous ethanol. In cultures of dissociated rat hippocampal neurons, these compounds have been shown to promote neuronal survival and to increase the number of branching points per axon (6). The chronic dietary intake of a low dose of SAC (40 mg/ $\mathrm{kg}$ ) has been reported to ameliorate deficits in learning performance and memory consolidation normally observed in the senescence-accelerated mouse strains, SAMP8 and SAMP10 (7). Recently, Baluchnejadmojarad et al demonstrated that chronic oral treatment with SAC $(150 \mathrm{mg} / \mathrm{kg})$ ameliorated cognitive deficits in a rat model of streptozotocin-induced diabetes by suppressing oxidative stress and neuroinflammation (8). Despite the accumulating knowledge of the properties of SAC in the presence of oxidative stress, little is known as to whether SAC ameliorates stress conditions other than oxidative stress, as well as the underlying mechanisms.

The endoplasmic reticulum (ER) is the largest organelle of most eukaryotic cells and plays crucial roles in various cellular processes, such as the synthesis, transport and correct folding of proteins, as well as lipid synthesis, carbohydrate metabolism and intracellular $\mathrm{Ca}^{2+}$ storage. Various physiological and pathological conditions, such as glucose deprivation, $\mathrm{Ca}^{2+}$ depletion and exposure to free radicals lead to the accumulation of misfolded or unfolded proteins in the ER, a condition known as ER stress (9). ER stress has been implicated in various neurological diseases, including brain ischemia, traumatic brain injury, and age-associated neurodegeneration. ER stress-induced cell death has been shown to be involved in various neurodegenerative diseases, such as Alzheimer's disease, Parkinson's disease, amyotrophic lateral sclerosis and Huntington's disease (10). Thus, it is possible that a pharmacological agent targeting the intracellular signaling of ER stress could provide a potential therapeutic approach for neurodegeneration and brain damage. The purpose of the present review was to summarize the neuroprotective mechanisms of $\mathrm{SAC}$ in relation to ER stress and to discuss the prospects of SAC becoming the prototype of a new type of therapeutic drug for neurodegenerative diseases linked to ER stress.

\section{Protective effects of SAC against neuronal death due to ER stress}

ER stress can be activated by various pathological and physiological conditions. The accumulation of misfolded or unfolded proteins in the ER activates a set of signaling pathways termed the unfolded protein response (UPR). The UPR can promote cellular repair and survival by reducing the load of unfolded proteins through the upregulation of chaperones and the attenuation of protein synthesis (11). However, when adaptive responses fail to resolve ER stress, the UPR ultimately initiates multiple, ER-specific pathways to induce apoptosis. In recent years, several lines of evidence have implicated several proteins in ER stress-induced cell death, such as $\mathrm{C} / \mathrm{EBP}$ homologous protein (CHOP), also known as growth arrest and DNA damage-inducible protein 153 (GADD153), caspase-12 and apoptosis-signal-regulating kinase 1 (ASK-1) (12). Among these stress-related factors, caspase-12 is localized specifically on the cytoplasmic side of the ER and has been shown to be proteolytically activated under conditions of ER stress in rodents $(13,14)$. Mutant mice lacking the caspase-12 gene are resistant to chemical ER stress inducers such as tunicamycin (an inhibitor of protein glycosylation), thapsigargin (an inhibitor of ER-associated $\mathrm{Ca}^{2+}$ ATPase) and brefeldin A (an inhibitor of ER-to-Golgi transport) (14). Previous studies by our group have demonstrated that neuronal death is mediated by caspase-12 when death is induced either by amyloid $\beta$-peptide (abbreviated $\mathrm{A} \beta$, and a key player in the pathology of Alzheimer's disease), or by tunicamycin, in both rat cultured hippocampal neurons $(15,16)$ and rat organotypic hippocampal slice cultures $(17,18)$. We have also previously demonstrated that the cell-permeable caspase-12-selective inhibitor, z-ATAD-fmk, significantly suppresses cell death induced by tunicamycin in organotypic hippocampal slice cultures $(17,18)$. Moreover, previous studies conducted in our laboratory have revealed that SAC protects against $\mathrm{A} \beta$ - and tunicamycin-induced cell death in 3 cell lines: PC12 cells differentiated by nerve growth factor (NGF) (19), cultured hippocampal neurons $(15,16,20)$ and organotypic hippocampal slice cultures $(17,18)$. Moreover, the increases in cleaved, activated caspase- 12 induced by $A \beta$ and tunicamycin have been shown to be prevented by the simultaneous application of SAC (15). The lipid peroxidation product, 4-hydroxynonenal, associated with oxidative stress, has been shown to play a pivotal role in the pathogenesis of a number of neurodegenerative disorders. However, SAC was shown to not prevent 4-hydroxynonenal-induced cell death in NGF-differentiated PC12 cells (19) or in cultured hippocampal neurons (15). Taken together, these results strongly suggest that SAC exerts a significant neuroprotective effect against ER stress-induced neuronal death by attenuating the activation of caspase-12.

\section{Calpain is a probable target molecule for SAC}

Three main mechanisms for caspase-12 activation by ER stress signals have been discovered thus far. First, caspase-12 forms a stable complex in the ER membrane with inositol-requiring enzyme 1 (IRE1) and the adapter protein tumor necrosis factor receptor-associated factor 2 (TRAF2) (21). The dissociation of TRAF2 from caspase-12 is reportedly a trigger for the activation of caspase-12 under ER stress conditions (21). Second, Rao et al have reported that in the 293T cell line, caspase-7 translocates from the cytosol to the ER membrane in response to ER stress, which leads to the processing of procaspase-12 and activation of caspase-12 (22). Third and most importantly, calpain, a cytoplasmic cysteine protease, has also been shown to cleave and activate caspase-12, which it does in response to $\mathrm{Ca}^{2+}$ release from the ER during ER stress (13). Furthermore, embryonic fibroblasts derived from conditional calpain knockout mice have been shown to be resistant to ER stress-induced cell death, which is due to resistance to caspase-12 activation (23). Moreover, the calpain inhibitor, PD150606, has been shown to inhibit tunicamycin-induced cell death in the kidney cell line, LLC-PK1 (24). These results suggest that the calpain activation pathway dominates in caspase-12-dependent, ER stress-induced cell death.

To further support this concept, we previously evaluated the effects of SAC on ER stress-induced calpain activation in cultured hippocampal neurons (20). Calpain activity can be measured by the quantitative analysis of full-length $\alpha$-spectrin, a neuron-specific cytoskeletal protein, which undergoes 


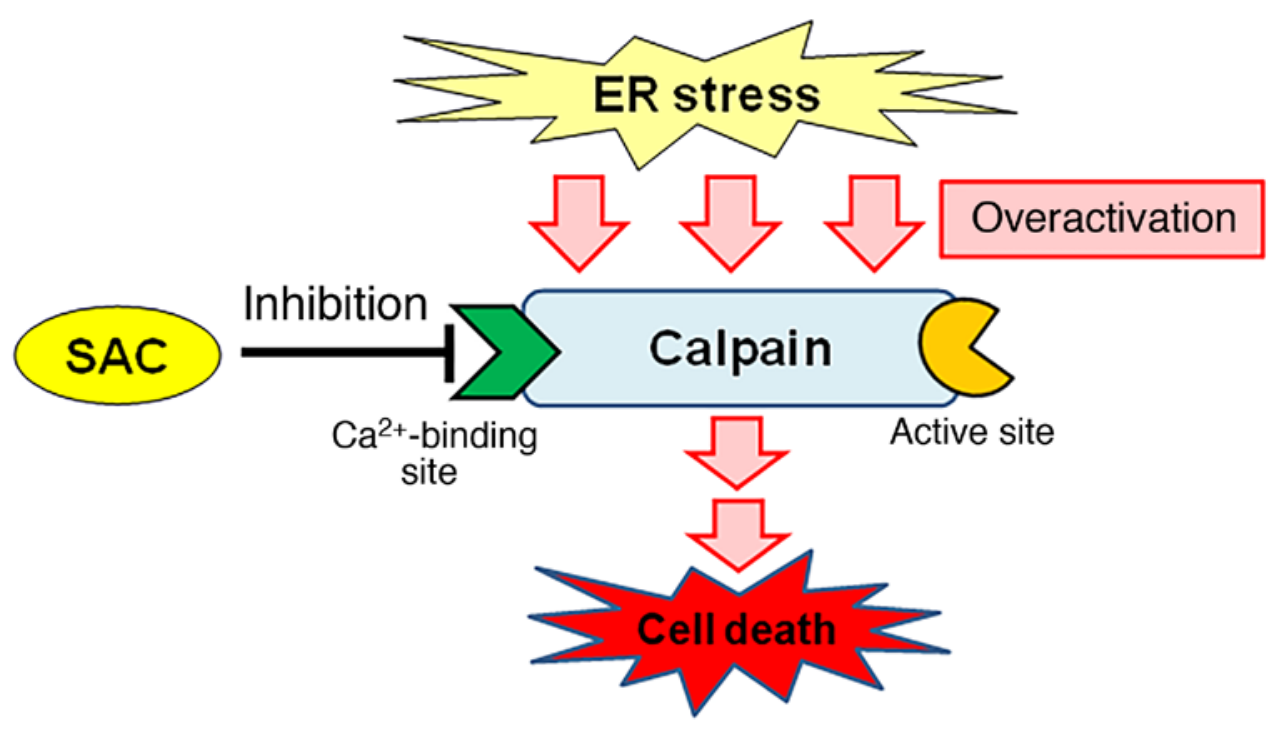

Figure 1. The proposed protective mechanism of $S$-allylcysteine (SAC) in endoplasmic reticulum (ER) stress-induced neuronal death in the hippocampus.

proteolysis by activated calpain. The exposure of cultured hippocampal neurons to tunicamycin for $24 \mathrm{~h}$ was shown to result in a decrease in the levels of full-length $\alpha$-spectrin. The degradation of $\alpha$-spectrin was significantly prevented by simultaneous treatment with SAC (20). We have also previously examined the effects of SAC on activation of recombinant calpain in a cell-free assay system containing a synthetic substrate for calpain, Suc-LLVY-Glo (20). The calpain activity was inhibited by SAC in a concentration-dependent manner, as observed with typical synthetic calpain inhibitors such as calpeptin, ALLN and PD150606 (20). At the higher concentrations tested (ALLN, $10 \mathrm{nM}$; PD150606, $100 \mathrm{mM}$ ), these synthetic calpain inhibitors completely inhibited calpain activity in our in vitro assay system. SAC partially inhibited calpain activity at still higher concentrations (10 mM) (20). These findings suggest that, at least in the hippocampus, the neuroprotective effects of SAC against ER stress are partly attributable to the direct inhibition of calpain activity.

To characterize the site of interaction of SAC with calpain, we previously examined the interactions of SAC with synthetic calpain inhibitors that have known calpain interaction sites: ALLN, which interferes with the active site of calpain, and PD150606, which interacts with the $\mathrm{Ca}^{2+}$-binding site $(20,25,26)$. When submaximal concentrations of synthetic calpain inhibitors were applied in the in vitro assay system, ALLN (5 pM) in combination with SAC additively inhibited calpain activity, whereas the inhibitory effect of PD150606 $(25 \mu \mathrm{M})$ was unaffected (20). By contrast, at supramaximal concentrations of synthetic calpain inhibitors, SAC significantly reversed the inhibitory effects of PD150606 $(100 \mu \mathrm{M})$, but did not affect inhibition by ALLN (1 nM) (20). Taken together, these results suggest that $\mathrm{SAC}$ interacts with the $\mathrm{Ca}^{2+}$-binding site of calpain (Fig. 1). This finding of a novel target for SAC strongly suggests that the limited concept of SAC as a mere antioxidant agent must be expanded to include the property of SAC of inhibiting ER stress. Although further in vivo studies should be carried out to clarify the underlying mechanisms responsible for the neuroprotective effects of SAC, the calpain inhibitory potential of SAC may prove to be useful in deriving therapeutic agents for neurological disorders associated with ER stress or the overactivation of calpain.

\section{Analogs of SAC}

In addition to SAC, various other sulfur-substituted compounds are abundant in the genus, Allium (27). Among these are $S$-methyl-L-cysteine (SMC), $S$-ethyl-L-cysteine (SEC) and $S$-propyl-L-cysteine (SPC), three typical hydrophilic cysteine-containing compounds naturally found in garlic (27). More recently, we synthesized various structurally related derivatives of SAC and examined their neuroprotective effects in a model of ER stress based on tunicamycin-induced neurotoxicity in rat cultured hippocampal neurons (28). The following $20 \mathrm{SAC}$ derivatives were tested: SAC itself, $S$-allyl-D-cysteine (ent-SAC), $S$-allyl-L-cysteine amide (SACA), $S$-benzyl-L-cysteine (SBC), $S$-benzyl-D-cysteine (ent-SBC), $S$-carboxymethyl-L-cysteine (SCMC), SEC, $S$-allyl-DL-homocysteine ( $r a c-S A H C), S M C$, SPC, $S$-propynyl-L-cysteine (SPNC), $S$-trityl-L-cysteine (STC), methyl $S$-allyl-L-cysteinate (MSAC), methyl $O$-allyl-L-serinate (MOAS), methyl $O$-allyl-N-boc-L-serinate (MOANBS), $N$-acetyl- $S$-allyl-L-cysteine (NASAC), $N$-acetyl- $S$-allyl-L-cysteine amide (NASACA), $O$-allyl-L-serine (OAS) and $O$-allyl-L-serine amide (OASA). Cultured hippocampal neurons were treated with tunicamycin $(10 \mu \mathrm{g} / \mathrm{ml})$ in the absence or presence of $1 \mu \mathrm{M}$ of a SAC derivative. These experiments demonstrated that the neuroprotective effects of the side-chain substituted SAC derivatives, SEC and SPC, against ER stress-induced cytotoxicity were more potent than those of SAC (Fig. 2). By contrast, compounds with bulky substituents (SBC, ent-SBC, SCMC, SPNC and STC) exerted neurotoxic effects. We have also previously demonstrated that several compounds with modified carboxyl or amino groups (SACA, rac-SAHC and NASAC) are as effective as SAC. Unlike SAC, SEC and SPC did not block calpain activity in the cell-free assay system, suggesting that the mechanism underlying the protective activity of SEC and SPC differs from that of SAC. We have also demonstrated that tunicamycin-induced cell death in cultured hippocampal neurons is not mediated 


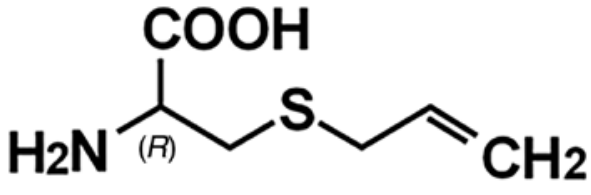

S-allyl-L-cysteine (SAC)

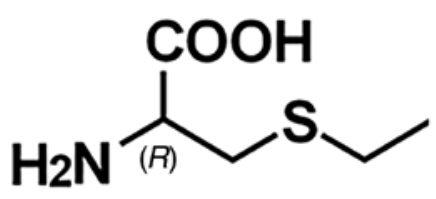

S-ethyl-L-cysteine (SEC)

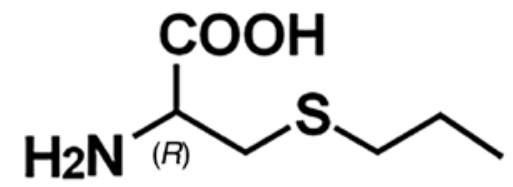

S-propyl-L-cysteine (SPC)

Figure 2. The chemical structures of the SAC derivatives.

by the oxidative stress cascade (20). Thus, we are left with the possibility that in hippocampal neurons, the stimulation of survival cascades other than the antioxidant defensive system underlies the neuroprotective action of SAC derivatives, such as SEC and SPC. Although further detailed studies are required to clarify the underlying mechanisms, it now appears that the number of carbon atoms in the linear 3-alkylthio groups of some SAC derivatives is key in potentiating neuroprotective effects against ER stress.

\section{Conclusions and future perspectives}

The pharmacokinetics of SAC are characterized by high oral absorption, limited metabolism, and extensive renal reabsorption, all of which potentially contribute to its high oral bioavailability (29). Moreover, SAC has already been used in the treatment of patients with hypertension without any obvious signs of toxicity (30). These results support the hypothesis that SAC may be a useful therapeutic agent with few harmful effects. Our group, as well as others have shown that SAC has neuroprotective potency in in vivo and in vitro in models of brain injury. SAC has been shown to ameliorate the neuronal damage usually observed in a rat model of transient middle cerebral artery occlusion (MCAO) (31). In a transgenic mouse model of Alzheimer's disease, SAC was shown to prevent synaptic degeneration (32) and the abnormal phosphorylation of tau protein (33). The dietary intake of SAC, SEC and SPC has been shown to decrease the production of $\mathrm{A} \beta$ in the brains of mice with D-galactose-induced aging (34). Similar cysteine-containing compounds exert neuroprotective effects against the loss of dopaminergic neurons in the 1-methyl-4-phenyl-1,2,3,6-tetrahydropyridine (MPTP) mouse model of Parkinson's disease $(35,36)$. Oxidative stress plays a critical role in the pathogenesis of the neurodegenerative diseases modeled above, including Alzheimer's disease,
Parkinson's disease and cerebral ischemic insults. However, a growing body of evidence indicates that oxidative stress has a strong connection with ER stress (37). Importantly, intracellular $\mathrm{Ca}^{2+}$ is a prime candidate for mediating the crosstalk between ER stress and oxidative stress. The surprising finding that calpain is a therapeutic target for SAC strongly suggests that SAC is not merely an antioxidant, but is also an inhibitor of ER stress signaling. Given the probable existence of intracellular $\mathrm{Ca}^{2+}$-mediated, ER-oxidative stress crosstalk, the fact that SAC blocks the calpain calcium-sensing site appears therapeutically advantageous. Moreover, the two-pronged activity profile appears ideally suited to treating a wide spectrum of neurodegenerative diseases in which both types of stress play a role. Finally, the chemical modification of the parent molecule has potential for achieving desirable changes in the pharmacodynamic profile. Although further in vivo detailed studies culminating in a clinical study are required, SAC and its derivatives, in their roles as both antioxidants and modulators of ER stress, can now be identified as good candidate drugs for the treatment of patients with neurodegenerative disorders. This concise review may provide new insight into the therapeutic potency of garlic-derived organosulfur compounds and may lead to the development of novel treatment strategies for patients with otherwise intractable neurological disorders.

\section{Acknowledgements}

The author would like to thank Editage (www.editage.com) for English language editing.

\section{Funding}

This study was supported in part by JSPS KAKENHI [Grant nos. JP26460634 and JP17K08965], by a Nihon University Chairman of the Board of Trustees Grant, and by a 'Private University Research Branding Project' from MEXT. The funding bodies had no role in the design of the study or in the writing of the manuscript.

\section{Availability of data and materials}

Not applicable.

\section{Authors' contributions}

YK designed the review, collected the information, drafted the manuscript, and finalized the manuscript. The author has read and approved the final manuscript.

\section{Ethics approval and consent to participate}

Not applicable.

\section{Patient consent for publication}

Not applicable.

\section{Competing interests}

The author declares that he has no competing interests. 


\section{References}

1. Colín-González AL, Santana RA, Silva-Islas CA, ChánezCárdenas ME, Santamaría A and Maldonado PD: The antioxidant mechanisms underlying the aged garlic extract- and $S$-allylcysteine-induced protection. Oxid Med Cell Longev 2012: 907162, 2012.

2. Saravanan G and Ponmurugan P: Antidiabetic effect of $S$-allylcysteine: Effect on thyroid hormone and circulatory antioxidant system in experimental diabetic rats. J Diabetes Complications 26: 280-285, 2012.

3. Yeh YY and Liu L: Cholesterol-lowering effect of garlic extracts and organosulfur compounds: Human and animal studies. J Nutr 131 (Suppl 3): 989S-993S, 2001.

4. Thomson $\mathrm{M}$ and Ali M: Garlic [Allium sativum]: A review of its potential use as an anti-cancer agent. Curr Cancer Drug Targets 3: 67-81, 2003.

5. Kodai S, Takemura S, Minamiyama Y, Hai S, Yamamoto S, Kubo S, Yoshida Y, Niki E, Okada S, Hirohashi K, et al: $S$-allyl cysteine prevents $\mathrm{CCl}(4)$-induced acute liver injury in rats. Free Radic Res 41: 489-497, 2007.

6. Moriguchi T, Matsuura H, Kodera Y, Itakura Y, Katsuki H, Saito H and Nishiyama N: Neurotrophic activity of organosulfur compounds having a thioallyl group on cultured rat hippocampal neurons. Neurochem Res 22: 1449-1452, 1997.

7. Nishiyama N, Moriguchi T,Morihara N and Saito H: Ameliorative effect of $S$-allylcysteine, a major thioallyl constituent in aged garlic extract, on learning deficits in senescence-accelerated mice. J Nutr 131 (Suppl 3): 1093S-1095S, 2001.

8. Baluchnejadmojarad T, Kiasalari Z, Afshin-Majd S, Ghasemi Z and Roghani M: $S$-allyl cysteine ameliorates cognitive deficits in streptozotocin-diabetic rats via suppression of oxidative stress, inflammation, and acetylcholinesterase. Eur J Pharmacol 794: 69-76, 2017.

9. Paschen W: Endoplasmic reticulum: A primary target in various acute disorders and degenerative diseases of the brain. Cell Calcium 34: 365-383, 2003

10. Placido AI, Pereira CM, Duarte AI, Candeias E, Correia SC, Carvalho C, Cardoso S, Oliveira CR and Moreira PI: Modulation of endoplasmic reticulum stress: An opportunity to prevent neurodegeneration? CNS Neurol Disord Drug Targets 14 518-533, 2015

11. Schröder M and Kaufman RJ: The mammalian unfolded protein response. Annu Rev Biochem 74: 739-789, 2005.

12. Gorman AM, Healy SJ, Jäger R and Samali A: Stress management at the ER: Regulators of ER stress-induced apoptosis. Pharmacol Ther 134: 306-316, 2012.

13. Nakagawa T and Yuan J: Cross-talk between two cysteine protease families. Activation of caspase-12 by calpain in apoptosis. J Cell Biol 150: 887-894, 2000.

14. Nakagawa T, Zhu H, Morishima N, Li E, Xu J, Yankner BA and Yuan J: Caspase-12 mediates endoplasmic-reticulum-specific apoptosis and cytotoxicity by amyloid-beta. Nature 403: 98-103, 2000.

15. Kosuge Y, Koen Y, Ishige K, Minami K, Urasawa H, Saito H and Ito Y: $S$-allyl-L-cysteine selectively protects cultured rat hippocampal neurons from amyloid beta-protein- and tunicamycin-induced neuronal death. Neuroscience 122: 885-895, 2003.

16. Kosuge Y, Sakikubo T, Ishige K and Ito Y: Comparative study of endoplasmic reticulum stress-induced neuronal death in rat cultured hippocampal and cerebellar granule neurons. Neurochem Int 49: 285-293, 2006.

17. Imai T, Kosuge Y, Ishige K and Ito Y: Amyloid beta-protein potentiates tunicamycin-induced neuronal death in organotypic hippocampal slice cultures. Neuroscience 147: 639-651, 2007.

18. Kosuge $\mathrm{Y}$, Imai $\mathrm{T}$, Kawaguchi M, Kihara T, Ishige $\mathrm{K}$ and Ito $\mathrm{Y}$ : Subregion-specific vulnerability to endoplasmic reticulum stress-induced neurotoxicity in rat hippocampal neurons. Neurochem Int 52: 1204-1211, 2008.

19. Ito $Y$, Kosuge Y, Sakikubo T, Horie K, Ishikawa N, Obokata N, Yokoyama E, Yamashina K, Yamamoto M, Saito $\mathrm{H}$, et al: Protective effect of $S$-allyl-L-cysteine, a garlic compound, on amyloid beta-protein-induced cell death in nerve growth factor-differentiated PC12 cells. Neurosci Res 46: 119-125, 2003.
20. Imai T, Kosuge $Y$, Endo-Umeda $K$, Miyagishi $H$, Ishige $K$, Makishima M and Ito Y: Protective effect of $S$-allyl-L-cysteine against endoplasmic reticulum stress-induced neuronal death is mediated by inhibition of calpain. Amino Acids 46: 385-393, 2014.

21. Yoneda T, Imaizumi K, Oono K, Yui D, Gomi F, Katayama T and Tohyama M: Activation of caspase-12, an endoplastic reticulum (ER) resident caspase, through tumor necrosis factor receptor-associated factor 2-dependent mechanism in response to the ER stress. J Biol Chem 276: 13935-13940, 2001

22. Rao RV, Hermel E, Castro-Obregon S, del Rio G, Ellerby LM, Ellerby HM and Bredesen DE: Coupling endoplasmic reticulum stress to the cell death program. Mechanism of caspase activation. J Biol Chem 276: 33869-33874, 2001.

23. Tan Y, Dourdin N, Wu C, De Veyra T, Elce JS and Greer PA: Ubiquitous calpains promote caspase-12 and JNK activation during endoplasmic reticulum stress-induced apoptosis. J Biol Chem 281: 16016-16024, 2006.

24. Muruganandan S and Cribb AE: Calpain-induced endoplasmic reticulum stress and cell death following cytotoxic damage to renal cells. Toxicol Sci 94: 118-128, 2006

25. Carragher NO: Calpain inhibition: A therapeutic strategy targeting multiple disease states. Curr Pharm Des 12: 615-638, 2006.

26. Wang KK, Nath R, Posner A, Raser KJ, Buroker-Kilgore M, Hajimohammadreza I, Probert AW Jr, Marcoux FW, Ye Q, Takano E, et al: An alpha-mercaptoacrylic acid derivative is a selective nonpeptide cell-permeable calpain inhibitor and is neuroprotective. Proc Natl Acad Sci USA 93: 6687-6692, 1996.

27. Jones MG, Hughes J, Tregova A, Milne J, Tomsett AB and Collin HA: Biosynthesis of the flavour precursors of onion and garlic. J Exp Bot 55: 1903-1918, 2004.

28. Imai T, Kosuge $Y$, Saito H, Uchiyama T, Wada T, Shimba S, Ishige K, Miyairi S, Makishima $M$ and Ito $Y$ : Neuroprotective effect of $S$-allyl-l-cysteine derivatives against endoplasmic reticulum stress-induced cytotoxicity is independent of calpain inhibition. J Pharmacol Sci 130: 185-188, 2016.

29. Amano H, Kazamori D and Itoh K: Pharmacokinetics of $S$-Allyl-1-cysteine in rats is characterized by high oral absorption and extensive renal reabsorption. J Nutr 146: 456S-459S, 2016.

30. Ried K, Frank OR and Stocks NP: Aged garlic extract lowers blood pressure in patients with treated but uncontrolled hypertension: A randomised controlled trial. Maturitas 67: 144-150, 2010.

31. Atif F, Yousuf S and Agrawal SK: $S$-allyl L-cysteine diminishes cerebral ischemia-induced mitochondrial dysfunctions in hippocampus. Brain Res 1265: 128-137, 2009.

32. Ray B, Chauhan NB and Lahiri DK: Oxidative insults to neurons and synapse are prevented by aged garlic extract and $S$-allyl-L-cysteine treatment in the neuronal culture and APP-Tg mouse model. J Neurochem 117: 388-402, 2011.

33. Chauhan NB: Effect of aged garlic extract on APP processing and tau phosphorylation in Alzheimer's transgenic model Tg2576. J Ethnopharmacol 108: 385-394, 2006.

34. Tsai SJ, Chiu CP, Yang HT and Yin MC: $S$-allyl cysteine, $S$-ethyl cysteine, and $S$-propyl cysteine alleviate $\beta$-amyloid, glycative, and oxidative injury in brain of mice treated by D-galactose. J Agric Food Chem 59: 6319-6326, 2011.

35. Chen CM, Yin MC, Hsu CC and Liu TC: Antioxidative and anti-inflammatory effects of four cysteine-containing agents in striatum of MPTP-treated mice. Nutrition 23: 589-597, 2007.

36. García E, Santana-Martínez R, Silva-Islas CA, ColínGonzález AL, Galván-Arzate S, Heras Y, Maldonado PD, Sotelo J and Santamaría A: $S$-allyl cysteine protects against MPTP-induced striatal and nigral oxidative neurotoxicity in mice: Participation of Nrf2. Free Radic Res 48: 159-167, 2014.

37. Nakka VP, Prakash-Babu P and Vemuganti R: Crosstalk between endoplasmic reticulum stress, oxidative stress, and autophagy: potential therapeutic targets for acute CNS injuries. Mol Neurobiol 53: 532-544, 2016.

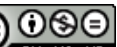

This work is licensed under a Creative Commons Attribution-NonCommercial-NoDerivatives 4.0 International (CC BY-NC-ND 4.0) License. 